\title{
Cirurgia de catarata realizada por residentes: avaliação dos riscos
}

\section{Cataract surgery performed by residents: risk analysis}

Jackson Barreto Junior; Helio Primiano Junior²; Rodrigo França de Espíndola ${ }^{3}$; Renato Antunes Schiave Germano $^{4}$, Newton Kara-Junior ${ }^{5}$

\begin{abstract}
ResUMO
Objetivo: Avaliar a frequência de complicações nas cirurgias de catarata realizada por residentes de um hospital universitário (segundo e terceiro anos), comparado com as realizadas por cirurgiões experientes (assistentes). Métodos: Análise retrospectiva dos prontuários de todos pacientes submetidos à cirurgia de catarata realizadas nas primeiras quinzenas de março (época do início do aprendizado da técnica cirúrgica) e de novembro (meados do aprendizado da técnica). Foram analisados a época da realização da cirurgia; graduação do cirurgião (residente ou médico assistente); técnica cirúrgica empregada (extração extracapsular ou facoemulsificação) e a ocorrência de complicações per-operatórias e pós-operatórias. Resultados: Foram analisadas 481 cirurgias, destas, 194 (40\%) foram realizadas pelos residentes do terceiro ano, $165(34 \%)$ pelos residentes do segundo ano e 116 (26\%) pelos assistentes. A complicação mais frequentemente encontrada em todas as cirurgias foi a rotura de cápsula posterior $(4,8 \%)$. Não houve diferença estatisticamente significativa de complicações entre as cirurgias realizadas em março e novembro ( $\mathrm{p}=0,97)$, bem como entre os residentes sob supervisão e os assistentes $(\mathrm{p}=0,08)$. Conclusão: A rotura de cápsula posterior continua sendo a complicação mais frequentemente encontrada nas cirurgias de residentes em treinamento. Não houve diferença estatisticamente significativa entre as taxas de complicação destes residentes e os assistentes, o que demonstra o importante papel de uma supervisão adequada.
\end{abstract}

Descritores: Facoemulsificação; Catarata; Internato e residência; Complicações intraoperatórias

\footnotetext{
'Doutor em Oftalmologia pela Universidade de São Paulo - USP - São Paulo (SP) - Brasil;

${ }^{2}$ Pós-graduando (Doutorado) pela Universidade de São Paulo - USP - São Paulo (SP) - Brasil;

${ }^{3}$ Estagiário do Setor de Catarata da Faculdade de Medicina da Universidade de São Paulo - FMUSP - São Paulo (SP) - Brasil;

${ }^{4}$ Acadêmico do quinto ano de graduação da Faculdade de Medicina da Universidade de São Paulo - FMUSP - São Paulo (SP) - Brasil;

${ }^{5}$ Livre-docente, Professor Colaborador da Faculdade de Medicina da Universidade de São Paulo, Chefe do Setor de Catarata do

Hospital das Clínicas da Faculdade de Medicina da Universidade de São Paulo - USP - São Paulo (SP), Brasil.

Trabalho realizado no Hospital das Clínicas da Faculdade de Medicina da Universidade de São Paulo - USP- São Paulo (SP) - Brasil.
}

Recebido para publicação em: 27/4/2010 - Aceito para publicação em 12/7/2010 


\section{INTRODUÇÃO}

A catarata é responsável por $50 \%$ da cegueira no mundo, com um número estimado de 20 milhões de casos que necessitariam de cirurgia ${ }^{(1)}$. Com o avanço das técnicas cirurgicas, sua correção nos dias de hoje é considerada um dos mais seguros e eficazes procedimentos ambulatoriais ${ }^{(2)}$.

Os programas de residência médica fazem parte do currículo de hospitais universitários. Com a disseminação do uso da facoemulsificação, o ensino desta técnica vem sendo inserida no programa de todos os principais centros oftalmológicos do mundo ${ }^{(3-4)}$.

As taxas de complicações durante a facoemulsificação realizadas por residentes variam na literatura de 2,0 a $14,7 \%{ }^{(4-5)}$. A perda vítrea continua sendo a mais indesejada destas complicações, e estudos mostram que, olhos com perda vítrea não atingem acuidade visual esperada em muitos casos ${ }^{(6-7)}$.

Com o objetivo de formar mais cirurgiões habilitados, e incentivados pela necessidade e pelo estímulo à realização de maior número de cirurgias de catarata no Brasil, muitos hospitais universitários elevaram o número de médicos residentes e aumentaram a capacidade de seus centros cirúrgicos ${ }^{(8)}$. Devido a este aumento, poderia se temer que as taxas de complicações operatórias se elevariam, e que a qualidade do serviço prestado não seria adequada.

O objetivo deste estudo é avaliar em um hospital universitário a frequência de complicações em cirurgias de catarata, realizadas por médicos em diferentes estágios de treinamento, quando comparados aos procedimentos realizados por cirurgiões experientes.

\section{MÉTODOS}

Trata-se de um estudo retrospectivo em que foram avaliados todos os prontuários das cirurgias de catarata realizadas no centro cirúrgico ambulatorial de um hospital universitário nas primeiras quinzenas dos meses de março (época do início do aprendizado da técnica cirúrgica) e de novembro (meados do aprendizado da técnica cirúrgica).

As cirurgias foram realizadas por 3 grupos de cirurgiões: 10 médicos assistentes do serviço de oftalmologia com experiência cirúrgica; 16 residentes do segundo ano (R2); e 12 residentes do terceiro ano (R3).

Os R2 utilizaram a técnica da extração extracapsular manual (ECCE), seguida pelo implante de lente intraocular (LIO) rígida de polimetil- metacrilato. Os R3 e os médicos assistentes utilizaram a técnica de facoemulsificação (FACO), seguida pelo implante de LIO acrílica hidrofóbica dobrável. Para a FACO foi utilizado o facoemulsificador modelo Legacy (Alcon Labs). Para ambas as técnicas cirúrgicas foi utilizado microscópio Zeiss III. Todas as cirurgias realizadas pelos residentes contaram com a supervisão de assistentes da clínica oftalmológica.

Foram estudadas as seguintes variáveis: época da realização da cirurgia (março ou novembro); graduação do cirurgião (residente ou assistente); técnica cirúrgica empregada (ECCE ou FACO) e a ocorrência de eventos adversos intraoperatórios e pós-operatórios imediatos.

Os dados foram analisados pelo programa estatístico - EpiInfo 2002. O teste estatístico utilizado foi o qui-quadrado com correção de Yates para intervalo de confiança de $95 \%$.

\section{Resultados}

Foram avaliadas no total 481 cirurgias de catarata, sendo que destas, 196 (40\%) foram realizadas por R3 (FACO), 167 (34\%) por R2 (ECCE), e 118 (26\%) por médicos assistentes (FACO).

Observou-se a ocorrência de 46 (9,6\%) complicações intra-operatórias e pós-operatórias imediatas (Tabela 1).

Quando foi comparada a ocorrência de complicações em diferentes períodos, observou-se que das 278 cirurgias realizadas em março, houve 26 (9,3\%) complicações. Em novembro ocorreram 20 (9,8\%) eventos adversos, de um total de 203 cirurgias. Não houve diferença estatística $(0,97)$ entre os dois períodos (Tabela 2).

Com relação a frequência de eventos adversos nas cirurgias realizadas pelos R2, observou-se que das 89 ECCE realizadas em março, houve $9(10,1 \%)$ casos com complicações, enquanto que no mês de novembro houve $8(10,5 \%)$ complicações em 76 cirurgias. Não houve diferença estatística $(\mathrm{p}=0,86)$ entre os dois períodos (Tabela 3).

Quanto à frequência de eventos adversos nas cirurgias realizadas pelos R3, observou-se que das 129 cirurgias realizadas em março houve $13(10,0 \%)$ casos com complicações, enquanto que no mês de novembro houve $10(15,4 \%)$ complicações em 65 cirurgias. Não houve diferença estatística $(\mathrm{p}=0,39)$ entre os dois períodos (Tabela 4).

Considerando-se o total de cirurgias realizadas, houve $40(11,1 \%)$ casos com complicações de um total de 359 cirurgias realizadas pelos residentes, e $6(5,1 \%)$ 
Tabela 1

Eventos adversos nas cirurgias de catarata realizadas pelos 3 grupos de cirurgiões

(R2*, R3** e assistentes)

\begin{tabular}{lcc}
\hline Eventos & N & \% \\
\hline Ruptura de cápsula posterior com perda vítrea & 23 & 4,8 \\
Ruptura de cápsula posterior sem perda vítrea & 12 & 2,5 \\
Luxação de fragmentos de cristalino para o vítreo & 03 & 0,6 \\
Diálise de zônula & 03 & 0,6 \\
Incisão anteriorizada & 02 & 0,4 \\
Sangramento de íris & 01 & 0,2 \\
Seidel no primeiro pós-operatório & 01 & 0,2 \\
Endoftalmite & 01 & 0,2 \\
\hline
\end{tabular}

(*) R2 = Residentes do segundo ano; (**) R3 = Residentes do terceiro ano

Tabela 2

Frequência total de complicações nas cirurgias realizadas nos meses de março e novembro

\begin{tabular}{lcccc}
\hline Período & \multicolumn{2}{c}{$\begin{array}{c}\text { Cirurgias com } \\
\text { complicaçóes }\end{array}$} & \multicolumn{2}{c}{ Cirurgias sem } \\
& Nomplicaçóes \\
N & \% & N & \% \\
\hline Março & 26 & 9,4 & 252 & 90,6 \\
Novembro & 20 & 9,9 & 183 & 90,1 \\
\hline
\end{tabular}

$\mathrm{P}=0,97 ; \mathrm{OR}=0,94 ; \mathrm{IC} 95 \%=0,49$ a 1,82

Tabela 4

Frequência de complicações nas cirurgias realizadas

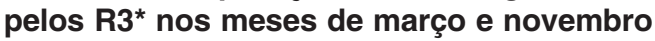

\begin{tabular}{lcccc}
\hline Período & \multicolumn{2}{c}{$\begin{array}{c}\text { Cirurgias com } \\
\text { complicações }\end{array}$} & \multicolumn{2}{c}{ Cirurgias sem } \\
& $\mathbf{N}$ & complicações \\
Março & 13 & 10,0 & N & \% \\
\hline Novembro & 10 & 15,4 & 55 & 84,0 \\
\hline
\end{tabular}

$\mathrm{P}=0,39 ; \mathrm{OR}=0,62 ; \mathrm{IC} 95 \%=0,23$ a 1,$63 ;(*) \mathrm{R} 3=$ Residentes do terceiro ano
Tabela 3

Frequência de complicações nas cirurgias realizadas pelos R2* nos meses de março e novembro

\begin{tabular}{|c|c|c|c|c|}
\hline \multirow[t]{2}{*}{ Período } & \multicolumn{2}{|c|}{$\begin{array}{l}\text { Cirurgias com } \\
\text { complicações }\end{array}$} & \multicolumn{2}{|c|}{$\begin{array}{l}\text { Cirurgias sem } \\
\text { complicações }\end{array}$} \\
\hline & $\mathbf{N}$ & $\%$ & $\mathbf{N}$ & $\%$ \\
\hline Março & 9 & 10,1 & 80 & 89,9 \\
\hline Novembro & 8 & 10,5 & 68 & 89,5 \\
\hline
\end{tabular}

Tabela 5

Frequência de complicações entre residentes $\left(R^{*}{ }^{*}\right.$ e $\left.R 3^{* *}\right)$ e médicos assistentes

\begin{tabular}{|c|c|c|c|c|}
\hline \multirow[t]{2}{*}{ Período } & \multicolumn{2}{|c|}{$\begin{array}{l}\text { Cirurgias com } \\
\text { complicações }\end{array}$} & \multicolumn{2}{|c|}{$\begin{array}{l}\text { Cirurgias sem } \\
\text { complicações }\end{array}$} \\
\hline & $\mathbf{N}$ & $\%$ & $\mathbf{N}$ & $\%$ \\
\hline Residentes & 40,0 & 11,1 & 319 & 88,9 \\
\hline Assistentes & 6 & 5,1 & 110 & 94,9 \\
\hline
\end{tabular}

$\mathrm{P}=0,23 ; \mathrm{OR}=0,76 ; \mathrm{IC} 95 \%=0,56$ a 1,$23 ;(*) \mathrm{R} 2=$ Residentes do segundo ano; $(* *) \mathrm{R} 3=$ Residentes do terceiro ano 
casos complicados em 116 cirurgias realizadas pelos assistentes. Não houve diferença estatística $(p=0,23)$ entre os 2 grupos (Tabela 5).

\section{DisCUSSÃo}

O ensino da técnica cirúrgica exige uma série de condições tais como: centro cirúrgico devidamente equipado e disponibilidade de cirurgiões capacitados para supervisionar o treinamento.Estudos mostram que dentre outros fatores, o sucesso da cirurgia de catarata em hospitais onde há residentes em treinamento consiste em uma supervisão feita por cirurgiões experientes ${ }^{(9)}$. Porém, mesmo em uma unidade cirúrgica equipada e com supervisão adequada, algumas cirurgias apresentam complicações, podendo limitar a acuidade visual pós-operatória ${ }^{(10,11)}$.

Considera-se que alguns dos principais fatores determinantes para a ocorrência de eventos adversos sejam o treinamento do cirurgião e a técnica utilizada ${ }^{(8,12)}$.

A literatura considera a ruptura de cápsula posterior com perda vítrea o evento adverso mais frequente, variando de 1,8 a $10,3 \%$ dos casos ${ }^{(13-18)}$. Em nosso estudo esta complicação também foi a mais frequentemente observada $(4,8 \%)$.

Em relação à técnica cirúrgica, muitos estudos sugerem que a técnica de facoemulsificação, quando realizada por cirurgiões experientes, seja mais segura do que a ECCE ${ }^{(19)}$. Entretanto, para residentes em treinamento, alguns estudos demonstraram que as taxas de perda vítrea são maiores durante a FACO ${ }^{(20,22)}$.

Nesta pesquisa, observou-se que ambas as técnicas cirúrgicas foram igualmente seguras, quando realizadas por cirurgiões em treinamento sob supervisão, comparadas com as cirurgias realizadas pelos cirurgiões experientes ( $\mathrm{p}=0,23)$. Outro estudo também demonstrou que quando os cirurgiões iniciantes são bem supervisionados o índice de complicações cirúrgicas não varia significativamente em relação a cirurgiões experientes ${ }^{(15)}$

A frequência de complicações ocorridas neste estudo com os R3 foi maior no final do treinamento do que no início. Esta diferença, embora estatisticamente não significativa, pode ser atribuída ao fato de que em geral, os residentes estão mais autoconfiantes e procuram operar casos com maior grau de dificuldade ao longo do ano. Como na FACO existem mais variáveis relacionadas ao cristalino que podem interferir na cirurgia, em relação a ECCE, como a consistência do núcleo lenticular, a liquefação do vítreo e o grau de dilatação pupilar, a cirurgia nestes casos, muitas vezes, está relacionada à maior taxa de complicações.
A incidência total de complicações entre os R3 durante o aprendizado da FACO foi de $13,4 \%$, número semelhante ao encontrado na literatura ${ }^{(4-5,23-25)}$.

\section{Conclusão}

Nas condições deste estudo, um cirurgião iniciante é capaz de realizar a cirurgia com segurança semelhante a um cirurgião experiente, desde que supervisionado por um cirurgião capacitado e disponha de tecnologia adequada. Assim, na maioria dos hospitais universitários, em que atualmente se dispõe de equipamentos cirúrgicos modernos e de um corpo clínico com cirurgiões capacitados e dispostos a ensinar, a qualidade da cirurgia de catarata oferecida à comunidade deve apresentar uma taxa similar de sucesso do que em instituições privadas.

\section{Abstract}

Purpose: To evaluate the complication's rate of cataract surgery performed by ophthalmology residents (second and third-year) and experienced surgeons at a public teaching hospital. Methods: A retrospective chart review of all patients who had cataract surgery between March (begin of the technique practice) and November (end of the technique practice) was conducted. Results: In 481 cataracts surgeries, 194 (40\%) was performed by thirdyear residents, 165 (34\%) by second-year residents and $116(26 \%)$ by experienced surgeons. The most frequent complication in all surgeries was the posterior capsule rupture $(4,8 \%)$. No statistical diference was found between the residents and experienced surgeons complication's rates ( $p=0,08)$. Conclusion: The posterior capsule rupture remains the most frequent complication during the cataract surgery learning curve. In this study there was no statistical difference between residents and experienced surgeons, which demonstrates the important role of adequate supervision of the surgeires.

Keywords: Phacoemulsification; Cataract; Internship and residency; Intraoperative complications

\section{RefERÊNCIAS}

1. Management of functional impairment due to cataract in adults. Cataract Management Guideline Panel. Ophthalmology. 1993;100(8 Suppl):1S-350S.

2. Schwab L. Cataract. In: Schwab L. Eye care in developing nations. 3rd ed. San Francisco: Foundation of the American Academy of Ophthalmology 1999. p. 21-52. 
3. Smith JH. Teaching phacoemulsification in US ophthalmology residencies: can the quality be maintained? Curr Opin Ophthalmol. 2005;16(1):27-32.

4. Unal M, Yücel I, Sarici A, Artunay O, Devranoglu K, Akar Y, Altin M. Phacoemulsification with topical anesthesia: Resident experience. J Cataract Refract Surg. 2006;32(8):13615. Comment in: J Cataract Refract Surg. 2007;33(5):755; author reply 755-6.

5. Bhagat N, Nissirios N, Potdevin L, Chung J, Lama P, Zarbin MA, et al. Complications in resident-performed phacoemulsification cataract surgery at New Jersey Medical School. Br J Ophthalmol. 2007;91(10):1315-7.

6. Frost NA, Sparrow JM, Strong NP, Rosenthal AR. Vitreous loss in planned extracapsular cataract extraction does lead to a poorer visual outcome. Eye (Lond). 1995;9(Pt 4):446-51.

7. Javitt JC, Vitale S, Canner JK, Street DA, Krakauer H, McBean AM, Sommer A. National outcomes of cataract extraction. Endophthalmitis following inpatient surgery. Arch Ophthalmol. 1991;109(8):1085-9.

8. Kara-Junior N, Arieta CEL. Catarata senil. In: Kara-José N, Almeida GV, editores. Senilidade ocular. São Paulo: Roca; 2001.

9. Quillen DA, Phipps SJ. Visual outcomes and incidence of vitreous loss for residents performing phacoemulsification without prior planned extracapsular cataract extraction experience. Am J Ophthalmol. 2003;135(5):732-3.

10. Pearson PA, Owen DG, Van Meter WS, Smith TJ. Vitreous loss rates in extracapsular cataract surgery by residents. Ophthalmology. 1989;96(8):1225-7.

11. Prakash G, Jhanji G, Sharma N, Gupta K, Titiyal JS, Vajpayee RB. Assessment of perceived difficulties by residents in performing routine steps in phacoemulsification surgery and in managing complications. Can J Ophthalmol. 2009;44(3):284-7.

12. Dantas PEC, Dantas MCN, Mandia Junior C, Waiswol M, Krasilchik G, Dias AKG. Facoemulsificação: a experiência da conversão; análise dos primeiros casos. Arq Bras Oftalmol. 1995;58(6):421-4.

13. Blomquist PH, Rugwani RM. Visual outcomes after vitreous loss during cataract surgery performed by residents. J Cataract Refract Surg. 2002;28(5):847-52.

14. Corey RP, Olson RJ. Surgical outcomes of cataract extractions performed by residents using phacoemulsification. J Cataract Refract Surg. 1998;24(1):66-72.

15. Tarbet KJ, Mamalis N, Theurer J, Jones BD, Olson RJ. Complications and results of phacoemulsification performed by residents. J Cataract Refract Surg. 1995;21(6):661-5.
16. Sappenfield DL, Driebe WT Jr. Resident extracapsular cataract surgery: results and a comparison of automated and manual techniques. Ophthalmic Surg. 1989;20(9):619-24.

17. Kara-José Junior N, Avakian A, Lower LMT, Rocha AM, Cursino M, Alves MR. Facoemulsificação versus extração extracapsular manual do cristalino: análise de custos. Arq Bras Oftalmol. 2004;67(3):481-9.

18. Santhiago MR, Monica LAM, Kara-Junior N, Gomes BAF, Bertino PM, Mazurek MGG, et al. Perfil do paciente com ceratopatia bolhosa pós-facectomia atendidos em hospital público. Rev Bras Oftalmol. 2009;68(4):201-5.

19. Albanis CV, Dwyer MA, Ernest JT. Outcomes of extracapsular cataract extraction and phacoemulsification performed in a university training program. Ophthalmic Surg Lasers. 1998;29(8):643-8.

20. Noecker RJ, Allinson RW, Snyder RW. Resident phacoemulsification experience using the in situ nuclear fracture technique. Ophthalmic Surg. 1994;25(4):216-21. Comment in: Ophthalmic Surg. 1995;26(1):92.

21. Allinson RW, Metrikin DC, Fante RG. Incidence of vitreous loss among third-year residents performing phacoemulsification. Ophthalmology. 1992;99(5):726-30. Comment in: Ophthalmology. 1992;99(8):1181. Ophthalmology. 1992;99(10):1481-2.

22. Prasad S. Phacoemulsification learning curve: experience of two junior trainee ophthalmologists. J Cataract Refract Surg. 1998;24(1):73-7.

23. Tarbet KJ, Mamalis N, Theurer J, Jones BD, Olson RJ. Complications and results of phacoemulsification performed by residents. J Cataract Refract Surg. 1995;21(6):661-5.

24. Tabandeh H, Smeets B, Teimory M, Seward H. Learning phacoemulsification: the surgeon-in-training. Eye (Lond). 1994;8(Pt 4):475-7.

25. Thomas R, Naveen S, Jacob A, Braganza A. Visual outcome and complications of residents learning phacoemulsification. Indian J Ophthalmol. 1997;45(4):215-9.

\section{Endereço para correspondência: \\ Praça das Hortências, $\mathbf{n}^{0} 70$ - Cond. Portal de Itu \\ CEP 13301-689. Itu - São Paulo (SP), Brasil \\ Tel: (11)4022-7273 \\ E-mail:rodrigo166@uol.com.br}

